\title{
RemoveYoung: A tool for the removal of the young stellar component in galaxies within an adjustable age cutoff
}

\author{
J. M. Gomes and P. Papaderos
}

\begin{abstract}
Instituto de Astrofísica e Ciências do Espaço, Universidade do Porto, Centro de Astrofísica da Universidade do Porto, Rua das Estrelas, 4150-762 Porto, Portugal

e-mail: jean@astro.up.pt
\end{abstract}

Received 15 February 2016/ Accepted 23 March 2016

\begin{abstract}
The optical morphology of galaxies holds the cumulative record of their assembly history, and techniques for its quantitative characterization offer a promising avenue toward understanding galaxy formation and evolution. However, the morphology of star-forming galaxies is generally dictated by the youngest stellar component, which can readily overshine faint structural/morphological features in the older underlying stellar background (e.g., relics from recent minor mergers) that could hold important insights into the galaxy build-up process. Stripping off galaxy images from the emission from stellar populations younger than an adjustable age cutoff $t_{\text {cut }}$ can therefore provide a valuable tool in extragalactic research. RemoveYoung $(\mathcal{R} \mathcal{Y})$, a publicly available tool that is presented here, exploits the combined power of integral field spectroscopy (IFS) and spectral population synthesis (SPS) toward this goal. Twodimensional (2D) post-processing of SPS models to IFS data cubes with $\mathcal{R} \mathcal{Y}$ permits computation of the spectral energy, surface brightness, and stellar surface density distribution of stellar populations older than a user-defined $t_{\text {cut }}$. This suggests a variety of applications of star-forming galaxies, such as interacting or merging galaxy pairs and lower mass starburst galaxies near and far; these include blue compact and tidal dwarf galaxies.
\end{abstract}

Key words. galaxies: starburst - galaxies: dwarf - galaxies: star clusters: general - galaxies: photometry - galaxies: stellar content galaxies: evolution

\section{Introduction}

How the assembly history of galaxies is imprinted on their present-day optical morphology is one of the most tantalizing enigmas in extragalactic research. Morphology holds the cumulative record of complex and highly interlinked processes operating on different temporal and spatial scales across cosmic time, such as quasi-monolithic gas collapse into classical bulges and galaxy spheroids, gentle gas dissipation into galactic disks, hierarchical growth via minor and major mergers, and environmentally modulated star formation (SF) in galaxy pairs/groups and clusters (see Kormendy \& Kennicutt 2004, for a review). Various quantitative morphology indicators (QMIs; see Conselice 2014, for a review) have been proposed in recent decades and extensively employed for the characterization of large extragalactic probes in the quest of elucidating the link between morphology, structure, intrinsic physical properties (e.g., stellar mass $\mathcal{M}_{\star}$ and surface mass density $\Sigma_{\star}$, metallicity) and the evolutionary and dynamical status of galaxies.

A first-order approximation in these studies is that the optical surface brightness $\mu$ traces the $\Sigma_{\star}$, which essentially presumes that the stellar mass-to-light ratio $(\mathcal{M} / \mathcal{L})$ spans a rather narrow range of values across the galaxy extent. Whereas this assumption is certainly justified for quiescent galaxies or systems with a smooth star formation history (SFH), it cannot be maintained for systems exhibiting a high specific star formation rate (sSFR), such as isolated and interacting starburst galaxies. The optical appearance of such galaxies primarily reflects the 2D distribution of the young ( $\$ 100 \mathrm{Myr})$ stellar component, which owing to its very low $\mathcal{M} / \mathcal{L}$ throughout overshines the older underlying stellar background that is dynamically dominant.

As an example, in a typical blue compact dwarf (BCD) galaxy the centrally confined starburst component dictates the observed line-of-sight intensity and contributes $50 \%$ to $80 \%$ of the total optical emission (Papaderos et al. 1996; Cairós et al. 2001; Gil de Paz \& Madore 2005). In BCDs and their higher- $z$ analogs (e.g., green peas; Cardamone et al. 2009; Izotov et al. 2011; Amorin et al. 2012, 2015) SF typically dominates down to $\mu \simeq 24.5-25.0 \mathrm{~B} \mathrm{mag} / \square^{\prime \prime}$, i.e., almost out to the Holmberg radius.

This strong disparity between $\mu$ and $\Sigma_{\star}$ in star-forming galaxies has a twofold effect: first, starburst emission can strongly impact light concentration indices (e.g., Morgan 1958; Abraham et al. 1996) commonly used in QMI studies. In a typical BCD, for instance, the ignition of a central starburst shrinks the optical effective radius by $\sim 70 \%$ (Papaderos et al. 2006) and can mimick a Sérsic profile with a high $(\eta=2-4)$ exponent (Papaderos et al. 1996; see also Bergvall \& Östlin 2002), eventually leading to its erroneous classification as an earlytype galaxy. Secondly, a tiny substrate of luminous young stars can readily masquerade fainter morphological features that potentially hold key insights into the recent assembly history and structural properties of galaxies (e.g., shells and ripples as relics from minor mergers; cf. Schweizer \& Seitzer 1988, or intrinsically faint bars).

In light of such considerations, a technique permitting suppression from galaxy images of the luminosity contribution from stars younger than an adjustable age cutoff $t_{\text {cut }}$ appears especially useful. Such a tool would not only be valuable to studies 

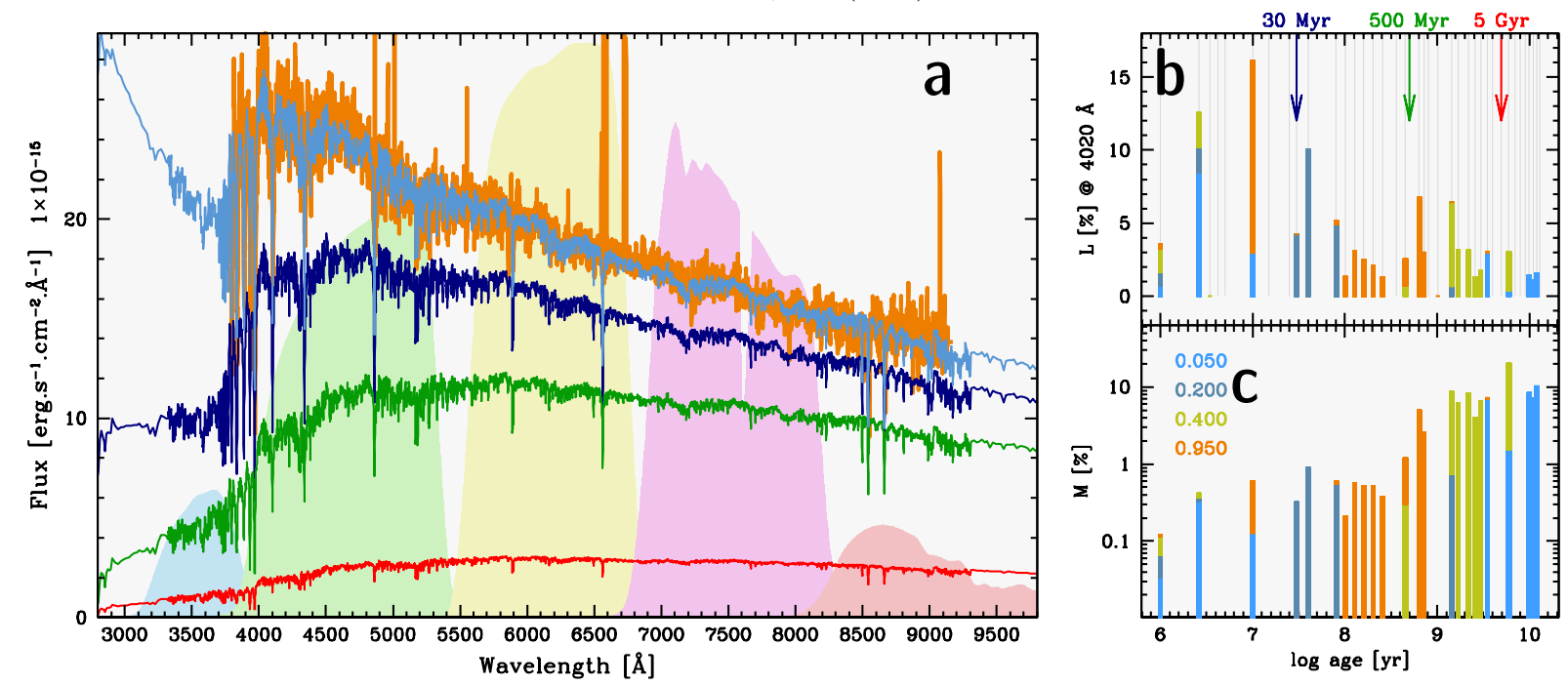

Fig. 1. a) Example of the application of $\mathcal{R} y$ to the best-fitting synthetic SED (light blue) obtained with STARLIGHT for a SDSS-DR7 (York et al. 2000) spectrum (orange) for three $t_{\text {cut }}$ values: $30 \mathrm{Myr}$ (blue), $0.5 \mathrm{Gyr}$ (green), and $5 \mathrm{Gyr}$ (red). The transmission curves of the five SDSS filters $u$, $g, r, i$, and $z$ are depicted with shaded areas. Panels $\mathbf{b})$ and $\mathbf{c}$ ) show, respectively, the luminosity $\left(x_{j}\right)$ and mass $\left(\mu_{j}\right)$ contribution (\%) of individual SSPs $(1 \ldots j)$ to the best-fitting population vector. The light-gray vertical lines in the upper-right panel show the ages available in the SSP library for four metallicities (between 0.05 and $0.95 Z_{\odot}$ ) and vertical arrows depict the applied $t_{\text {cut }}$ values. From panels b) and c) it is apparent that, according to the best-fitting population vector, SSPs younger than $30 \mathrm{Myr}$ contribute less than $2 \%$ of $\mathcal{M}_{\star}$ but nearly $35 \%$ of the observed intensity at the normalization wavelength of $4020 \AA$. As a result, suppression of these young SSPs results in a significant dimming by $0.56,0.27,0.22$, and 0.19 mag in the SDSS $u, g, r$, and $i$-bands, respectively.

of QMIs, but also to those exploring SF patterns back to a welldefined time interval (e.g., since the infall of a galaxy onto a cluster). This task is obviously out of reach with standard techniques, such as near-infrared (NIR) imaging (e.g., Noeske et al. 2003) or state-of-the-art modeling of the observed spectral energy distribution (SED; e.g., Wuyts et al. 2012).

In this article, we present a publicly available ${ }^{1}$ tool, RemoveYoung $(\mathcal{R} \mathcal{Y})$, which exploits the combined power of integral field spectroscopy (IFS) and spectral population synthesis (SPS) with the goal of stripping off galaxy images from the luminosity contribution from stellar populations younger than a user-defined age cutoff. The concept is outlined in Sect. 2 and illustrated through its application to IFS data in Sect. 2.1, with a discussion of its potential merits to various subjects of extragalactic research following in Sect. 3 .

\section{Concept and realization of $\mathcal{R} y$}

The concept of $\mathcal{R} \mathcal{Y}$ essentially consists in the elimination from a synthetic SED of the contribution from stellar populations younger than an adjustable age cutoff $t_{\text {cut }}$ and reconstruction of the residual SED of the older stellar component. The input SED is generally obtained through SPS modeling of an observed spectrum as a linear combination of simple stellar population (SSP) spectra, each fully characterizing the SED of an instantaneously formed stellar population of a given metallicity and age. The best-fitting population vector (PV) from a SPS model $M_{\lambda}$ encapsulates the contribution of individual SSPs along with the derived intrinsic extinction and stellar velocity dispersion. Mathematically, $\mathcal{R} \boldsymbol{Y}$ acts essentially as a "signal post-processing" tool for the recovered PV. The bestfitting model $M_{\lambda}$ is multiplied by the Heaviside unit step function $^{2} H\left(t-t_{\text {cut }}\right)$, permitting suppression from the SED of the

\footnotetext{
1 A thoroughly documented version of the code is available at http://www. spectralsynthesis.org

${ }^{2} H\left(t-t_{\text {cut }}\right)=\int_{-\infty}^{t-t_{\text {cut }}} \delta(s) \mathrm{d} s=0$ for $t<t_{\text {cut }}$ and 1 for $t \geq t_{\text {cut }}$.
}

contribution from stellar populations that are younger than an age cutoff $t_{\text {cut }}$ to the SED

$\mathcal{R} \mathcal{Y}_{\lambda}=M_{\lambda} H\left(t-t_{\text {cut }}\right)=M_{\lambda_{0}}\left[\sum_{j=1}^{N_{\star}} x_{j} b_{j, \lambda} r_{\lambda}\right] \otimes G\left(v_{\star}, \sigma_{\star}\right) H\left(t-t_{\text {cut }}\right)$,

where $b_{j, \lambda} \equiv L_{\lambda}^{\mathrm{SSP}}\left(t_{j}, Z_{j}\right) / L_{\lambda_{0}}^{\mathrm{SSP}}\left(t_{j}, Z_{j}\right)$ is the spectrum of the $j$ th SSP normalized at $\lambda_{0}, r_{\lambda} \equiv 10^{-0.4\left(A_{\lambda}-A_{\lambda_{0}}\right)}$ is the reddening term parametrized by the stellar extinction $A_{\lambda}$ at $\lambda, \boldsymbol{x}=$ $\left(x_{1}, \ldots, x_{N_{\star}}\right)$ is the population vector, $M_{\lambda_{0}}$ is the synthetic flux at the normalization wavelength, $N_{\star}$ is the total number of SSPs, and $G\left(v_{\star}, \sigma_{\star}\right)$ is the line-of-sight stellar velocity distribution modeled as a Gaussian centered at velocity $v_{\star}$ and broadened by $\sigma_{\star}$.

In the following, we use for the sake of demonstration bestfitting PVs from the SPS code STARLIGHT (Cid Fernandes et al. 2005). We note, however, that $\mathcal{R} \boldsymbol{Y}$ can be applied to the output from any other SPS code. Figure 1a illustrates the postprocessing with $\mathcal{R} \mathcal{Y}$ of a spectral model that is based on a library of 152 SSPs from Bruzual \& Charlot (2003) spanning an age between $1 \mathrm{Myr}$ and $13 \mathrm{Gyr}$ for four metallicities $(0.05,0.2$, 0.4 , and $\left.0.95 Z_{\odot}\right)$. Panels $\mathrm{b}$ and $\mathrm{c}$ show, respectively, the light and mass contribution (\%) of the library SSPs composing the PV. The structure of the latter, as a linear superposition of SSPs, facilitates straightforward reconstruction of the synthetic SED of the stellar component older than any age cutoff, as illustrated in panel a for three $t_{\text {cut }}$ values (30 Myr, $0.5 \mathrm{Gyr}$, and $5 \mathrm{Gyr}$ ).

Also, $\mathcal{R} \mathcal{Y}$ computes and exports several other quantities, such as the apparent magnitude of the stellar component with age $t \lessgtr t_{\text {cut }}$ in several standard broadband filters (Johnson-Cousins, Bessel, SDSS, 2MASS and others), with the provision of correction for intrinsic extinction. Further important features of $\mathcal{R} \mathcal{Y}$ are computation of UV-through-NIR broadband magnitudes from the best-fitting SED and hybrid observed+synthetic magnitudes through substitution of spectral intervals of a filter transmission curve eventually not covered by observations by the modeled spectrum; for example, estimation of the SDSS $i$-band magnitude from a low-resolution spectrum from the CALIFA IFS 
J. M. Gomes and P. Papaderos: RemoveYoung: A tool for the removal of the young stellar component in galaxies
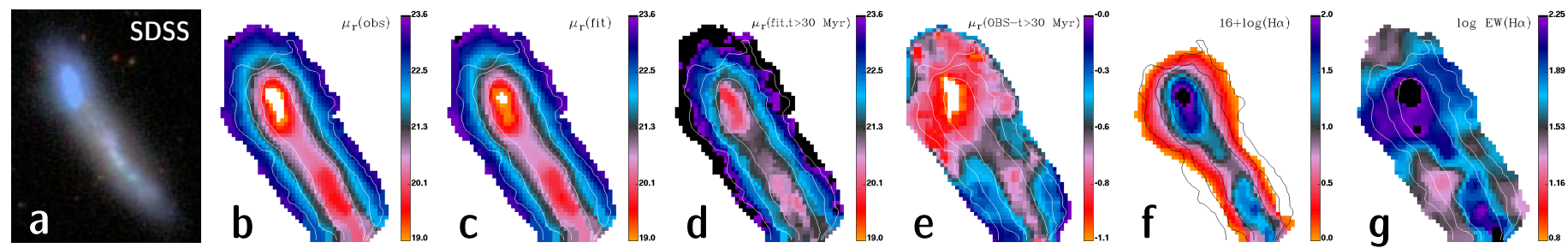

Fig. 2. Two-dimensional application of $\mathcal{R} \mathcal{Y}$ on CALIFA IFS data for the star-forming galaxy NGC 3991. a) True-color SDSS image composite; b)-d) surface brightness maps (mag/ $\left.\square^{\prime \prime}\right)$ computed by spaxel-by-spaxel convolution of the $r$-band filter transmission curve with the observed spectrum $\left(\mu_{\mathrm{obs}}\right.$; panel $\left.\mathbf{b}\right)$, the best-fitting stellar SED to the observed spectrum $\left(\mu_{\mathrm{fit}}\right.$; panel $\left.\mathbf{c}\right)$ ), and after removal with $\mathcal{R} \boldsymbol{Y}$ of stellar and nebular emission associated with ongoing or recent ( $\leq 30 \mathrm{Myr})$ star formation $\left(\mu(\mathrm{OBS}-30 \mathrm{Myr})\right.$; panel d)). Subtraction of the latter from $\mu_{\mathrm{obs}}$ yields the $\mu$ enhancement (in mag) owing to the recent SF (panel e)), which shows a close spatial correlation with the $\mathrm{H} \alpha$ flux (in $\log 10^{-16} \mathrm{erg} \mathrm{s}^{-1} \mathrm{~cm}^{-2}$; panel f)) and $\mathrm{H} \alpha$ equivalent width (in $\AA$; panel g)).
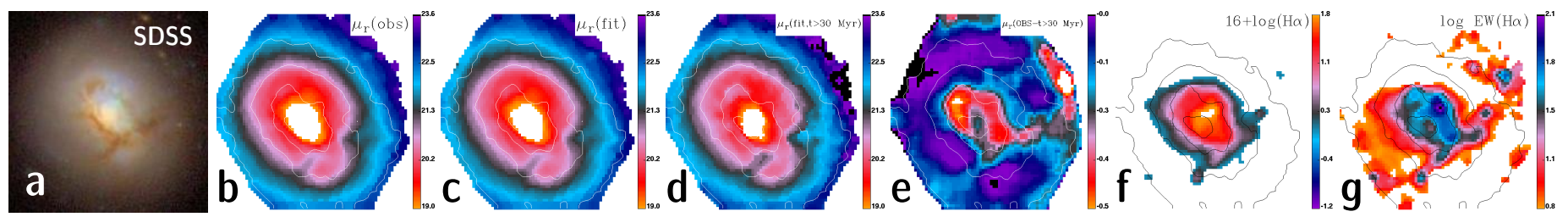

Fig. 3. Application of $\mathcal{R} y$ on CALIFA IFS data for the luminous BCD galaxy NGC 7625. The panels have the same meaning as in Fig. 2.

survey spanning a spectral range between $3745 \AA$ and truncated at $7300 \AA$. Besides the magnitudes and SEDs of stellar populations with $t \lessgtr t_{\text {cut }}, \mathcal{R} \mathcal{Y}$ also exports $\mathcal{M}_{\star}$, both prior to and after correction for the mass fraction returned into the ISM.

\subsection{Two-dimensional application of $\mathcal{R} y$ to IFS data cubes}

Two-dimensional applications of $\mathcal{R} \mathcal{Y}$ for stripping off the young stellar component from IFS data cubes are greatly aided by the structured format in which the computed quantities are stored. To demonstrate the 2D functionality of the code, we apply it next on two nearby SF galaxies from the second public data release (García-Benito et al. 2015) of the CALIFA galaxy survey (Sánchez et al. 2012). Both systems were initially processed with our automated IFS pipeline PORTO3D (Papaderos et al. 2013; Gomes et al. 2016a), which allows for spectral modeling with STARLIGHT and derivation of emission-line fluxes, equivalent widths (EWs), and kinematics. Fits were computed spaxel-byspaxel between $4000 \AA$ and $6800 \AA$ using the same SSP library as in Sect. 2.

The first galaxy, NGC 3391 ( $D=50.9$ Mpc; Fig. 2), hosts intense SF activity at the NE tip of an elongated cometary (also referred to as tadpole) host galaxy (cf. the true-color SDSS image composite in Fig. 2a) that manifests itself on a $g-i$ color $-0.1 \ldots$ $-0.5 \mathrm{mag}$ and an $E W(\mathrm{H} \alpha) \sim 300 \AA$ over a region of $\sim 2.5 \mathrm{kpc}$ in diameter. Spectral fits indicate that $\mathrm{SF}$ in that region has been ongoing since $\sim 10^{8} \mathrm{yr}$ with the young $\left(\leq 30 \mathrm{Myr} \equiv t_{\text {cut }, 1}\right)$, ionizing stellar component representing $\approx 4 \%$ of $\mathcal{M}_{\star}$. The synthetic surface brightness $\mu$ (mag/ $\left.\square^{\prime \prime}\right)$ maps in panels b and c were computed from the IFS data cubes by convolving the observed $\left(\mu_{\mathrm{obs}}\right)$ and modeled $\left(\mu_{\mathrm{fit}}\right)$ SED with the SDSS $r$-band filter transmission curve. Assuming for simplicity that nebular continuum emission is negligible (e.g., however, see Krüger et al. 1995; Papaderos et al. 1998), subtraction of $\mu_{\mathrm{fit}}$ from $\mu_{\mathrm{obs}}$ permits quantification of the luminosity enhancement $(\approx-0.2 \mathrm{mag}$, corresponding to $17 \%$ of the $r$-band line-of-sight intensity) due to nebular line contamination in the NE SF region. Panels d and e show, respectively, the reconstructed $\mu_{r}$ for the stellar component older than $30 \mathrm{Myr}$ and its difference to $\mu_{\mathrm{obs}}$. Much like in Fig. 1a, suppression of the young SF component (along with nebular emission) has a striking effect on the SED, revealing a complex $\mu_{r}$-enhancement pattern by $\sim 0.6$ mag all over the NE half of NGC 3991 and up to $-1.2 \mathrm{mag}$ in its off-center SF knot (panel e). As apparent from comparison with the $\mathrm{H} \alpha$ and $\operatorname{EW}(\mathrm{H} \alpha)$ maps (panels $\mathrm{f}$ and $\mathrm{g}$ ), there is a good spatial correspondence between the $\mu_{r}$ enhancement because of ongoing SF (panel e) and its nebular tracers, which adds circumstantial support to the soundness of the approach taken.

A second example of the 2D application of $\mathcal{R} y$ to IFS data is given in Fig. 3 for the luminous BCD NGC 7625 ( $D=23.7 \mathrm{Mpc}$, Thuan \& Martin 1981; Cairós et al. 2001, 2012). Panel d shows the $\mu_{r}$ map of the stellar component for $t \geq t_{\text {cut, } 1}$ with panel $\mathrm{e}$ revealing a complex $\mu_{r}$ enhancement pattern reflecting the interplay between young stars, dust obscuration, and extended nebular emission (cf. panels $\mathrm{f}$ and $\mathrm{g}$ ).

With an increasing $t_{\text {cut }}, \mathcal{R} \mathcal{Y}$ uncovers a progressively smooth, lower surface brightness (LSB) elliptical host with a central $\mu_{r}$ of $19 \mathrm{mag} / \square^{\prime \prime}$ for a $t_{\text {cut }}=5$ Gyr. This value is 1.3 mag fainter than the observed $\mu$ and in excellent agreement with the results from $R$-band profile decomposition by Papaderos (1998). The potential of $\mathcal{R} \mathcal{Y}$ for surface photometry studies of star-forming galaxies can be better appreciated from panel a of Fig. 4, where we show with open circles the observed SDSS $r$-band surface brightness profile (SBP) of NGC 7625 together with synthetic $r$-band SBPs (solid circles) computed from the CALIFA IFS data cube for four $t_{\text {cut }}$ values. It can be seen that the synthetic SPB for $t_{\text {cut }}=0$ Gyr matches the observed SPB out to the maximum photometric radius $\left(R^{\star} \lesssim 30^{\prime \prime}\right)$ corresponding to the CALIFA field of view, except for its innermost $\left(\$ 3^{\prime \prime}\right)$ part because of the lower FWHM $(\sim 2 ! 7)$ of the IFS data. At $t_{\text {cut }}=0.3$ Gyr (blue symbols), the SBP suggests a flattening of the exponential intensity profile of the LSB host inward of $\sim 15^{\prime \prime}$, which might be reproduced by, for example, the modified exponential fitting function proposed in Papaderos et al. (1996) or a Sérsic profile with $\eta<1$. This pronounced intensity flattening at intermediate radii also implies that SF activity in NGC 7625 is not confined to the innermost part of the LSB host, as SDSS true-color images suggest, but is instead spatially extended over the central $\sim 30^{\prime \prime}$ of the BCD. Independent support of this conjecture comes from unsharp masking of SDSS $g, r$, and $i$ images, which reveals 


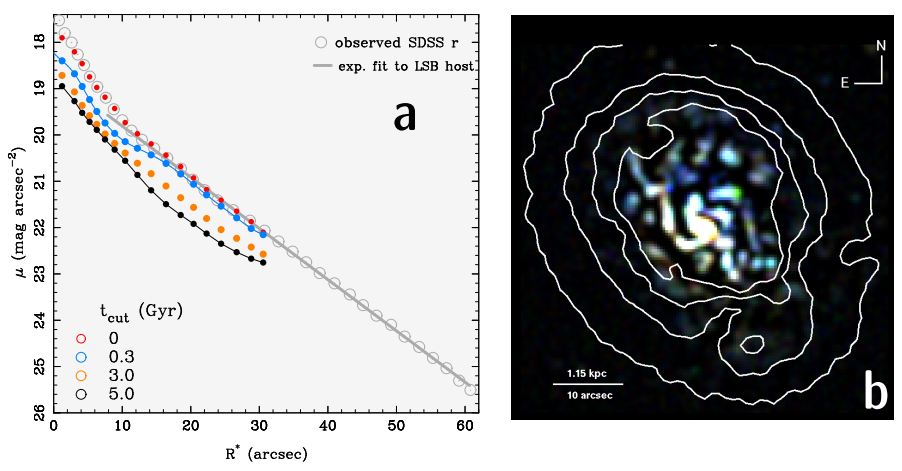

Fig. 4. a) Surface brightness profile (SBP) of NGC 7625 (open circles) computed from SDSS $r$-band data with the overlaid gray line showing a linear fit to the LSB host for $R^{\star} \geq 20^{\prime \prime}$. Filled circles show synthetic $r$-band SBPs obtained by applying $\mathcal{R} \boldsymbol{Y}$ to CALIFA IFS data for four $t_{\text {cut }}$ values $(0,0.3,3$, and 5 Gyr). b) True-color composite image computed by applying the flux-conserving unsharp masking technique by Papaderos et al. (1998) to the SDSS $g, r$, and $i$ images. The contours delineate the morphology of the emission-line free stellar continuum between $6390 \AA$ and $6490 \AA$, as obtained from CALIFA IFS data.

a complex pattern of multiple blue SF knots within the central $\sim 3.5 \mathrm{kpc}$ of NGC 7625 (panel b). Finally, stars older than $3 \mathrm{Gyr}$ and 5 Gyr (orange and black symbols, respectively) describe a nearly exponential distribution with a scale length close to that obtained from a linear fit to the observed SBP of the LSB host for $R^{\star} \geq 20^{\prime \prime}$ (straight gray line). It is interesting to note that the central surface brightness implied from the latter fit to observed data $\left(\sim 19 \mathrm{mag} / \square^{\prime \prime}\right)$ is consistent with the central $\mu_{r}$ read off the synthetic SBP for $t_{\text {cut }}=5 \mathrm{Gyr}$, a fact pointing to the additional potential of $\mathcal{R} \mathcal{Y}$ as a supportive tool to profile decomposition studies of star-forming galaxies.

\section{Discussion and summary}

Before providing an outline of potential applications of $\mathcal{R} \boldsymbol{Y}$, we offer some cautionary notes: Evidently, the output from $\mathcal{R} \mathcal{Y}$ sensitively relies on the quality and soundness of solutions from SPS models. These are known to suffer from a number of deficiencies, such as the notorious age-metallicity degeneracy, which may propagate into hardly predictable and as yet poorly explored biases in best-fitting SFHs. Additionally, the rather restricted number of library SSPs allowed by state-of-the-art SPS codes (at maximum 300 for STARLIGHT) permits a rather coarse coverage of the age and metallicity parameter space, which results in a strongly discretized approximation to the true SFH of a galaxy. Quite obviously, $\mathcal{R} \mathcal{Y}$ cannot offer a better time resolution than that of the SSP library used for the input SPS models. Consequently, $\mathcal{R} \mathcal{Y}$ can fully unfold its potential only in conjunction with next-generation SPS codes that are capable of significantly alleviating the above shortcomings.

Another aspect to bear in mind when interpreting the output from $\mathcal{R} \mathcal{Y}$ is that the detectability of morphological relics (e.g., past SF episodes) does not only depend on their spectrophotometric fading, but also on dynamical processes. For example, differential disk rotation acts to erase signatures of an accreted satellite within a few rotational periods $(\sim 250 \mathrm{Myr}$ for a MW-like system). Likewise, $\Sigma_{\star}$ enhancements due to minor mergers could gradually disperse in the presence of processes that eventually contribute to inside-out galaxy growth, such as bar-driven stellar migration (Sellwood \& Binney 2002; Berentzen et al. 2007; Roskar et al. 2008; Sánchez-Blázquez et al. 2014) in normal galaxies, diffusion of newly formed stars (Papaderos et al. 2002; Papaderos \& Östlin 2012) in dwarf galaxies, or outwardly propagating SF (Gomes et al. 2016b).

On the other hand, the briefly outlined applications of $\mathcal{R} \mathcal{Y}$ on IFS data (Sect. 2.1) illustrate the potential of the code in various fields of extragalactic research. For example, a natural byproduct of $\mathcal{R} \mathcal{Y}$ is the removal of nebular emission and the quantification of its effect on broadband photometry. Already, with a conservative $t_{\text {cut }}$ on the order of the main-sequence lifetime of ionizing stars (10-30 Myr), $\mathcal{R} \mathcal{Y}$ permits partial suppression of bright SF regions from synthetic images; this opens new avenues to structural and QMI studies of starburst galaxies where the young stellar component and nebular emission frequently dominate within the optical extent. Combined with deep IFS, $\mathcal{R} \mathcal{Y}$ may thus be regarded as an analogous yet more powerful approach to the structural properties of high-sSFR systems than NIR photometry. This is not only because the latter is expensive in terms of observational time and data reduction effort, but also because it per se does not permit complete suppression of stellar populations younger than an adjustable age cutoff. Additionally, the $K$-band $\mathcal{M} / \mathcal{L}$ of an instantaneously formed stellar population varies by $\sim 2$ dex within $100 \mathrm{Myr}$, depending on metallicity and initial stellar mass function, which implies that an accurate determination of $\mathcal{M}_{\star}$ requires even in NIR wavelengths prior knowledge of the SFH. The adjustable age cutoff allowed by $\mathcal{R} y$ is a key advantage here, which together with an extensive set of output quantities (e.g., $\mathcal{M}_{\star}$ and synthetic images for an unlimited number of broad- and narrowbands) suggests a broad range of applications. Examples follow.

i) Subtraction of the SF component from BCDs and other starburst galaxies, allowing for improved determinations of the central intensity distribution (and, henceforth, the gravitational potential) of the underlying host galaxy. An unresolved question in this context is whether the latter shows an extended flat core, which under certain assumptions would imply a central minimum in the radial stellar density distribution (Papaderos et al. 1996; see also Noeske et al. 2003). ii) Spatial progression of SF activity in galaxies: This subject encompasses several particular aspects, ranging from the hypothesis of unidirectional SF propagation in cometary galaxies (Papaderos et al. 1998, 2008), to the formation history of multiple generations of young stellar clusters (SCs) in isolated and interacting starburst galaxies (e.g., Östlin et al. 2003; Adamo et al. 2011; Whitmore et al. 2007), to the outward propagation of a SF front in collisional ring galaxies (Appleton \& Struck-Marcell 1996; Romano et al. 2008). Adaptive removal of SCs and/or a more diffusely distributed young stellar substrate for a set of increasing $t_{\text {cut }}$ can, in principle, provide a powerful technique for the reconstruction of SF propagation patterns and their role in galaxy build-up. iii) Tidal dwarf galaxy (TDG) formation: are these entities forming through gas collapse within a pre-existing gravitational potential from tidally ejected stars or out of a purely gaseous selfgravitating component? (e.g., Weilbacher et al. 2002; see also Duc 2012 for a review). Subtraction of the SF component with $\mathcal{R} \mathcal{Y}$ could add decisive constraints for discriminating between both scenarios. iv) Galaxy evolution in clusters: galaxies falling onto clusters may experience a complex SFH involving, for example, an initial starburst episode followed by ram-pressure induced cessation of SF (e.g., Poggianti et al. 1999) in some cases accompanied by kpc-long, UV-emitting SF tails (e.g., Hester et al. 2010; Kenney et al. 2014). $\mathcal{R} \boldsymbol{Y}$ offers a handy tool to explore galaxy evolution in clusters back to several $10^{8} \mathrm{yr}$, i.e., over the critical phase of ram-pressure stripping. v) Last but not least, a natural application of $\mathcal{R} \mathcal{Y}$ concerns robust determinations of QMI sets, such as CAS (concentration-asymmetry-smoothness; 
Conselice 2003) and the Gini coefficient (Lotz et al. 2004), after decontamination of IFS data cubes from SF or directly from $\Sigma_{\star}$ maps.

The above examples outline the potential and wide range of applications of $\mathcal{R} \mathcal{Y}$ toward deciphering the galaxy assembly history in the modern era of integral field spectroscopy.

Acknowledgements. We would like to thank the anonymous referee for valuable comments and suggestions. J.M.G. acknowledges support by Fundação para a Ciência e a Tecnologia (FCT) through the Fellowship SFRH/BPD/66958/2009 and POPH/FSE (EC) by FEDER funding through the Programa Operacional de Factores de Competitividade (COMPETE). P.P. is supported by FCT through the Investigador FCT Contract No. IF/01220/2013 and POPH/FSE (EC) by FEDER funding through the program COMPETE. JMG\&PP acknowledge support by the FCT under project FCOMP-01-0124-FEDER-029170 (Ref. PTDC/FISAST/3214/2012), funded by FCT-MEC (PIDDAC) and FEDER (COMPETE) and the Exchange Programme "Study of Emission-Line Galaxies with IntegralField Spectroscopy" (SELGIFS, FP7-PEOPLE-2013-IRSES-612701), funded by the EU through the IRSES scheme. This paper is based on data from the Calar Alto Legacy Integral Field Area Survey, CALIFA (http://califa. caha. es), funded by the Spanish Ministery of Science under grant ICTS-200910, and the Centro Astronómico Hispano-Alemán. This research made use of the NASA/IPAC Extragalactic Database (NED), which is operated by the Jet Propulsion Laboratory, California Institute of Technology, under contract with the National Aeronautics and Space Administration.

\section{References}

Abraham, R. G., Tanvir, N. R., Santiago, B. X., et al. 1996, MNRAS, 279, L47 Adamo, A., Östlin, G., Zackrisson, E., et al. 2011, MNRAS, 415, 2388

Amorín, R., Pérez-Montero, E., Vílchez, J. M., \& Papaderos, P. 2012, ApJ, 749, 185

Amorín, R., Pérez-Montero, E., Contini, T., et al. 2015, A\&A, 578, A105

Appleton, P. N., \& Struck-Marcell, C. 1996, Fundamentals of Cosmic Physics, 16,111

Berentzen, I., Shlosman, I., Martinez-Valpuesta, I. et al. 2007, ApJ, 666, 189

Bergvall, N., \& Östlin, G. 2002, A\&A, 390, 891

Bruzual, G., \& Charlot, S. 2003, MNRAS, 344, 1000

Cairós, L. M., Vílchez, J. M., González Pérez, J. N., et al. 2001, ApJS, 133, 321
Cairós, L. M., Caon, N., García Lorenzo, B., et al. 2012, A\&A, 547, A24 Cardamone, C., Schawinski, K., Sarzi, M., et al. 2009, MNRAS, 399, 1191 Cid Fernandes, R., Mateus, A., Sodré, L., Stasińska, G., \& Gomes, J. M. 2005, MNRAS, 358, 363

Conselice, C. J. 2003. ApJS 147, 1

Conselice, C. J. 2014, ARA\&A, 52, 291

Duc, P.-A. 2012, in Proc. JENAM 2010, eds. P. Papaderos, S. Recchi, \& G. Hensler (Lisbon: Springer Verlag), 188

García-Benito, R., Zibetti, S., Sánchez, S. F., et al. 2015, A\&A, 576, A135

Gil de Paz, A., \& Madore, B. F. 2005, ApJS, 156, 345

Gomes, J. M., Papaderos, P., Kehrig, C., et al. 2016a, A\&A, 588, A68

Gomes, J. M., Papaderos, P., Vílchez, J. M., et al. 2016b, A\&A, 586, A22

Hester, J. A., Seibert, M., Neill, J. D., et al. 2010, ApJ, 716, L14

Izotov, Y. I., Guseva, N., \& Thuan, T. X. 2011, ApJ, 728, 161

Kenney, J. D. P., Geha, M., Jáchym, P., et al. 2014, ApJ, 780, 119

Kormendy, J., \& Kennicutt, R. C. 2004, ARA\&A, 42, 603

Krüger, H., Fritze-v. Alvensleben, U., Loose, H.-H. 1995, A\&A, 303, 41

Lotz, J. M., Primack, J., \& Madau, P. 2004, AJ, 128, 163

Morgan, W. W. 1958, PASP, 70, 364

Noeske, K. G. Papaderos, P., Cairós, L. M., \& Fricke, K. J. 2003, A\&A, 410, 481

Östlin, G., Zackrisson, E., Bergvall, N., \& Rönnback, J. R. 2003, A\&A, 408, 887

Papaderos, P. 1998, Ph.D. Thesis, University of Göttingen

Papaderos, P., \& Östlin, G. 2012, A\&A, 537, A126

Papaderos, P., Loose, H.-H., Thuan, T. X., \& Fricke, K. J. 1996, A\&AS, 120, 207

Papaderos, P., Izotov, Y. I., Fricke, K. J., et al. 1998, A\&A, 338, 43

Papaderos, P., Izotov, Y. I., Thuan, T. X., et al. 2002, A\&A, 393, 461

Papaderos, P., Guseva, N. G., Izotov, Y. I., et al. 2006, A\&A, 457, 45

Papaderos, P., Guseva, N. G., Izotov, Y. I., \& Fricke, K. J. 2008, A\&A, 491, 113

Papaderos, P., Gomes, J. M., Vílchez, J. M., et al. 2013, A\&A, 555, L1 (P13)

Poggianti, B. M., Smail, I., Dressler, A., et al. 1999, ApJ, 518, 576

Romano, R., Mayya, Y. D., \& Vorobyov, E. I. 2008, AJ, 136, 1259

Roskar, R., Debattista, V. P., Stinson, G. S., et al. 2008, ApJ, 675, 65

Sánchez, S. F., Kennicutt, R. C., Gil de Paz, A., et al. 2012, A\&A, 538, A8

Sánchez-Blázquez, P., Rosales-Ortega, F. F., Méndez-Abreu, J., et al. 2014, A\&A, 570, A6

Schweizer, F., \& Seitzer, P. 1988, ApJ, 328, 88

Sellwood, J. A., \& Binney, J. J. 2002, MNRAS, 336, 785

Thuan, T. X., \& Martin, G. E. 1981, ApJ, 247, 823

Weilbacher, P. M., Fritze-v. Alvensleben, U., Duc, P.-A., \& Fricke, K. J. 2002, A\&A, 579, 79

Whitmore, B. C., Chandar, R., \& Fall, S. M. 2007, AJ, 133, 1067

Wuyts, S., Förster-Schreiber, N., Genzel, R., et al. 2012, ApJ, 753, 114

York, D. G., Adelman, J., Anderson, Jr., J. E., et al. 2000, AJ, 120, 1579 